\title{
Abandono escolar en educación media superior: conocimiento y aportaciones de política pública
}

Highschool drop-out: Knowledge and public policy contributions

\section{FRANCISCO MirAnda LÓPEZ*}

Este artículo tiene el objetivo de realizar un balance de la magnitud y las dimensiones del problema del abandono escolar en la educación media superior, con base en algunas aportaciones teóricas y la revisión de los principales programas gubernamentales y las acciones públicas que se han desarrollado en México. Orientados por una metodología cualitativa, basada en la revisión teórica, la recuperación de información estadística fundamental y la sistematización de experiencias de políticas y programas para abatir el abandono escolar en la EMS, generamos un marco analítico para ordenar y analizar las principales estrategias gubernamentales, así como las directrices emitidas por el Instituto Nacional para la Evaluación de la Educación. Este análisis arroja como resultado la presencia de una tendencia de las políticas públicas para atender factores de riesgo, principalmente socioeconómicos, pero ignora aspectos fundamentales como el ambiente y la integración escolar. Por ello, se proponen algunas recomendaciones para fortalecer la política pública en el ámbito educativo que reconocen la heterogeneidad de las causas del abandono.

This paper aims to make a balance of the magnitude and dimensions of the high school dropout, using some theoretical contributions and a review about the main Mexican policies. Oriented by a qualitative methodology, based on the theoretical review, the recovery of fundamental statistical information and the systematization of experiences of policies and programs oriented to the prevention of abandonment in the high school, an analytical framework was generated to organize and analyze the main government strategies, as well as the guidelines issued by the National Institute for the Evaluation of Education. The analysis finds a policy trend to address risk factors, mainly socioeconomic, but ignores fundamental issues such as the school environment and school integration. Therefore, some recommendations are proposed to strengthen public policy in the educational field, recognizing the heterogeneity of the causes of dropout.

\section{Palabras clave:}

abandono escolar, política pública, riesgo social, desafiliación educativa, des-subjetivación

\section{Keywords:}

school dropout, public policy, social risk, educational disaffiliation, des-subjectivation

Recibido: 28 de febrero de 2018. | Aceptado para su publicación: 16 de agosto de 2018. DOI: 10.31391/S2007-7033(2018)0051-010

\footnotetext{
* Doctor en Ciencias Sociales por El Colegio de México. Miembro del Sistema Nacional de Investigadores, nivel II. Titular de la Unidad de Normatividad y Política Educativa en el Instituto Nacional para la Evaluación de la Educación. Fue coordinador nacional de la Reforma de la Educación Secundaria de 2005 a 2007. Sus líneas de investigación abordan la educación, política educativa y formación docente.
} 


\section{INTRODUCCIÓN}

T a educación es un derecho humano fundamental, ya que es condición esencial y potenciadora del desarrollo de los demás derechos de las niñas, niños y adolescentes. En México, este derecho se reconoce en el artículo $3^{\circ}$ de la Constitución Política de los Estados Unidos Mexicanos y en leyes de carácter federal, como la Ley General de Educación, la Ley General de los Derechos de Niñas, Niños y Adolescentes y la Ley General de Desarrollo Social. En el ámbito internacional, existen diversos instrumentos jurídicos, entre los que destacan la Declaración Universal de los Derechos Humanos, la Convención sobre los Derechos del Niño y la Convención Iberoamericana de Derechos de los Jóvenes, los cuales reconocen y protegen el derecho de los niños, las niñas y los jóvenes a una educación gratuita, continua, libre y de calidad.

En 2008, mediante el acuerdo 442 emitido por la Secretaría de Educación Pública, se impulsó la Reforma Integral de la Educación Media Superior con la finalidad de mejorar los aprendizajes, modernizar el bachillerato general y administrar la diversidad de planes y programas de estudio entre los distintos subsistemas que configuran la educación media superior (EMS) (Diario Oficial de la Federación, 2008). En 2012 se estableció por decreto el mandato constitucional de la obligatoriedad de la EMS y se indicó el deber del Estado "... de ofrecer un lugar para cursarla a quien teniendo la edad típica hubiera concluido la educación básica..." (Diario Oficial de la Federación, 2012); se estableció como plazo para el cumplimento efectivo de esta obligación el ciclo escolar 2021-2022. Con ello se adhiere la EMS a la trayectoria de educación obligatoria y se da un paso importante en el reconocimiento del derecho a la educación.

La reforma educativa de 2013, con la intención no solo de expandir la cobertura de la educación, sino también de ofrecer servicios de calidad, promovió como eje la calidad del sistema educativo, definida como el máximo logro educativo de los estudiantes. Además, se hace depender la calidad educativa de la adaptación y mejora de otros componentes educativos relevantes, a saber: los materiales y métodos educativos, la organización escolar, la infraestructura educativa y la idoneidad de los docentes y directivos (art. $3^{\circ}$, párrafo 3, de la Constitución Política de los Estados Unidos Mexicanos, 1917).

Como advertimos, existe un marco normativo robusto que sostiene y promueve el derecho a una educación media superior de calidad y con equidad, cuyo cumplimiento efectivo puede mirarse cuando los adolescentes y jóvenes: i) acceden a una oferta educativa de calidad, con hincapié en aquellos que enfrentan condiciones desfavorables; ii) siguen trayectorias escolares sin rezago y egresan de acuerdo con la edad típica de terminación de los grados y niveles respectivos, en condiciones de oferta flexibles; y iii) adquieren los aprendizajes relevantes que responden a sus necesidades en contextos culturales y sociales diversos (Miranda e Islas, 2016).

Los mandatos de ley son condición necesaria, pero no son suficientes para garantizar el acceso, la permanencia y el logro máximo de los aprendizajes de los estudiantes en la EMS. Por ello, las acciones de política pública que se desarrollen en México son fundamentales para garantizar, en condiciones de equidad, el derecho al acceso, la permanencia y el logro educativo en la EMS. Así, por ejemplo, el Plan Nacional de Desarrollo 2013-2018 (Gobierno de la República, 2013) resalta la importancia de renovar el sistema educativo y subraya que, para lograr la cobertura universal con pertinencia en educación media superior (y superior), es necesario 
fortalecer las carreras de corte tecnológico y vincularlas al sector productivo. Además, destaca que los estudiantes desarrollen habilidades, competencias y capacidades que les permitan insertarse en el ámbito laboral.

Asimismo, en el Programa Sectorial de Educación 2013-2018 se asume la necesidad de la apertura de nuevas escuelas y mejora de las existentes, y se reconoce como aspecto fundamental minimizar el número de estudiantes que abandonan sus estudios en este tipo educativo. Para atender el problema de abandono en la EMS, plantea garantizar la calidad de los planes y programas de estudio que se ofrecen en cada plantel, desarrollar vínculos con el sector productivo, establecer una comunicación estrecha con los padres y madres de familia para que apoyen el proceso formativo de sus hijos e hijas, y reducir los factores de riesgo que influyen sobre la permanencia de los jóvenes en la escuela. Asimismo, considera prioritario atender el desarrollo profesional de los docentes (Secretaría de Educación Pública [SEP], 2013).

Sin embargo, pareciera que las diversas acciones de política pública tampoco han sido suficientes para resarcir la deuda social que el Estado mexicano tiene con la EMS, pues persisten las brechas en el acceso entre diversos grupos poblacionales; además, los estudiantes que pueden acceder no tienen las mejores condiciones para permanecer, lograr trayectorias educativas exitosas, egresar y concretar el aprendizaje de conocimientos y desarrollo de competencias relevantes, hecho que se refleja siempre en contra de los más desfavorecidos.

En este contexto normativo y político, nuestro objetivo es describir la magnitud y las dimensiones del problema del abandono escolar en la EMS en México a fin de proponer algunas aportaciones teóricas y experiencias internacionales exitosas a partir de las cuales se revisan los principales programas gubernamentales y acciones públicas que se han desarrollado en el país para enfrentar el abandono escolar. Al final, además de las conclusiones, proponemos algunas recomendaciones para fortalecer la política pública en la materia.

Para cumplir el objetivo planteado, optamos por una metodología cualitativa que, además de la recuperación de información estadística relevante, consistió en la revisión documental y el posterior análisis de planteamientos teóricos, informes de resultados de las políticas y los programas dirigidos a la prevención del abandono y atención a la permanencia escolar en la EMS.

\section{EL PROBLEMA PÚBLICO}

Analizamos los principales indicadores que dan cuenta de las características del problema público en la EMS, específicamente en las causas del abandono escolar.

Estructura de la EMS: fragmentación de los servicios

Uno de los rasgos en la composición de la EMS en México es su diversidad institucional y heterogeneidad curricular. De acuerdo con el artículo 46 de la Ley General de Educación, la EMS se estructura por las modalidades escolarizada, no escolarizada y mixta; se compone de tres modelos educativos: bachillerato general, bachillerato tecnológico y bachillerato profesional técnico; tiene distintos tipos de sostenimiento: administrados por las autoridades educativas federal y locales, universidades públicas, organismos públicos descentralizados y el sector privado. Asimismo, están 
organizados por cinco tipos de control administrativo y presupuestario: centralizado, descentralizado, desconcentrado, autónomo y privado (Instituto Nacional para la Evaluación de la Educación [INEE], 2017a). Además, se encuentran otros bachilleratos con tipos de formación diversa: “... el militar, el de artes y humanidades, el intercultural bilingüe, el integral comunitario, el incluyente y el Modelo Mexicano de Formación Dual, entre otros" (Dander, 2017, p. 82).

Lo anterior supone una diversidad no solo administrativa y curricular, sino también de los contextos socioeducativos que se presentan en este tipo educativo. Sin embargo, en los denominados subsistemas se dan inequidades en la calidad, acceso, permanencia y término de la trayectoria educativa de los jóvenes que cursan la EMS. Asimismo, la heterogeneidad de la EMS se ha traducido en fragmentación institucional y estratificación de los servicios educativos que se reflejan en la desvinculación entre los distintos sistemas y servicios existentes. Por ello, en 2008 se buscó dar respuesta a esta situación al articular los subsistemas a través del Sistema Nacional de Bachillerato y del Marco Curricular Común.

En la actualidad, el Modelo Educativo 2017 reconoce la existencia en la EMS de 33 subsistemas con 150 expresiones organizacionales e institucionales. Como parte de los planteamientos de este modelo, se creó el Sistema Nacional de Educación Media Superior, coordinado por la SEP. Este mecanismo articulador se orienta a la atención de los problemas de pertinencia y relevancia, y de la falta de equivalencias curriculares entre subsistemas, que responde a la heterogeneidad de estos.

El citado sistema, como mecanismo articulador, promueve el cumplimiento de estándares de calidad y pertinencia en los planes y programas de estudio, capacitación docente y directiva, infraestructura y equipamiento, con el objetivo de que los planteles de EMS cumplan con los requerimientos para ingresar al Padrón de Buena Calidad del Sistema Nacional de Bachillerato (SEP, 2017).

Además, se cuenta con el Espacio Común de la Educación Media Superior, entendido como un instrumento de gestión y comunicación para la cooperación e intercambio, desde el cual se desarrollan políticas comunes en el marco del respeto a la diversidad con la finalidad de mejorar los servicios educativos de las distintas instituciones de EMS.

A pesar de los esfuerzos realizados y los previstos a corto plazo, el denominado Sistema de Educación Media Superior sigue siendo un "archipiélago de subsistemas" con serias limitaciones para producir identidad, equidad y acciones afirmativas para una juventud crecientemente diferenciada y fracturada en su composición sociocultural.

\section{Brechas de acceso, permanencia y logro}

Desde la declaración de obligatoriedad de la educación media superior (en 2012) ha habido un incremento en la tasa de absorción, la cual pasó de 100.9 en el ciclo escolar 2012-2013 a 101.1 en 2015-2016. Debemos tener en cuenta, de acuerdo con el INEE (2017c), que este cálculo es sobreestimado debido a la presencia de estudiantes egresados de secundaria en generaciones anteriores que se incorporan en forma reciente o reingresan a este tipo educativo.

Para dar cuenta solo de los estudiantes que ingresan de inmediato al bachillerato una vez terminada la secundaria, recurrimos al trabajo de Solís (2018), que propone 
un nuevo cálculo para estimar cuántos de los egresados de secundaria del ciclo anterior en realidad ingresaron al siguiente nivel educativo en el ciclo sucesivo; obtuvimos una tasa de absorción estimada de $80.7 \%$ en 2015. Esto sugiere que no se está cumpliendo el derecho consignado en el acuerdo 442 para casi $20.0 \%$ de los jóvenes que tienen la edad típica y egresaron de manera oportuna de la educación básica.

Considerando a los adolescentes y jóvenes con edad idónea o típica, en el ciclo escolar 2015-2016 se matriculó 59.5\% de ellos en EMS, 6.6 puntos porcentuales más que los reportados en el ciclo escolar 2012-2013 (INEE, 2016).

Sin embargo, si bien se observan avances importantes en la ampliación de la cobertura educativa en la EMS, persisten problemas de abandono escolar y bajo logro educativo acompañados de brechas por tipo de sostenimiento, tamaño de localidad, grado de marginación, condición de indígena y situación socioeconómica.

La tasa de aprobación en educación media superior en el ciclo escolar 2014-2015 fue de $84.4 \%$, casi un punto porcentual arriba de la registrada en el ciclo escolar 2009-2010, que fue de 83.8\% (INEE, 2016). Por tipo de sostenimiento, el mayor porcentaje de aprobación lo reportaron las escuelas privadas sin subsidio (84.4\%), y en las escuelas con sostenimiento público fue de $83.2 \%$. El porcentaje de alumnos con extraedad grave en el ciclo escolar 2015-2016 fue de 13.5\%, y se reportó una disminución importante en comparación con el ciclo escolar 2010-2011, la cual fue de 14.1\% (INEE, 2016). Según el grado de marginación, en las escuelas urbanas de baja marginación se ubica el mayor porcentaje de extraedad grave (14.4\%).

Por lo que se refiere al logro académico, los resultados de la prueba PLANEA EMS son contundentes y preocupantes, porque una tercera parte de los estudiantes del tercer año de bachillerato (33.9\%) no alcanzan los niveles de aprendizaje esperado en lengua y comunicación, y la situación empeora en matemática, en la que $66.2 \%$ de los estudiantes tienen dificultades (INEE, 2017d).

Existe un problema generalizado de bajos niveles de aprendizaje, con brechas importantes: i) por control administrativo, los mejores resultados corresponden en ambos casos a los planteles de bachillerato de las universidades autónomas estatales, mientras que los resultados más bajos se obtienen en los planteles de control administrativo estatal; ii) por condición de habla de lengua indígena, los puntajes son significativamente menores en ambas áreas en aquellos estudiantes que señalaron contar con al menos un padre hablante de alguna lengua indígena; iii) por percentil económico, los mejores resultados los obtienen, en ambas disciplinas, los estudiantes que se ubican en los percentiles más altos (INEE, 2017c).

En el análisis de la situación de la EMS, sobresale un problema neurálgico, que es la tasa de abandono escolar. Para el ciclo escolar 2015-2016, esta fue de 15.5\%, que en términos absolutos equivale a cerca de setecientos mil jóvenes que abandonan los estudios cada año (INEE, 2017a). Si se observa por modelo educativo, el bachillerato general presenta la menor proporción de abandono (13.3\%), seguido por el bachillerato tecnológico (15.8\%); el profesional técnico presenta los mayores niveles de abandono (24.3\%) (INEE, 2017c). Por sostenimiento, en el bachillerato privado se reportaron las mayores tasas de abandono escolar (18.7\%), mientras que la menor se identificó en los bachilleratos de universidades autónomas, con 12.2\% (INEE, 2017a). 
Las causas asociadas al abandono escolar en la EMS son diversas y cada una tiene una naturaleza distinta. Los resultados de las investigaciones nacionales e internacionales (Abril, Román, Cubillas y Moreno, 2008; Attanasio \& Székely, 2003; Banco Interamericano de Desarrollo, 2012; Comisión Económica para América Latina y el Caribe, 2015; Goicovic, 2002; Huerta, 2010; INEE, 2017a), así como de diversas encuestas: Encuesta Nacional de Ocupación y Empleo 2009; Encuesta Nacional de la Juventud 2010; Encuesta Nacional de Deserción en la Educación Media Superior 2012) (Instituto Mexicano de la Juventud y SEP, 2010; Subsecretaría de Educación Media Superior, SEP, y Consejo para la Evaluación de la Educación del Tipo Medio Superior, 2012), indican que las principales razones de abandono escolar que reportan los adolescentes y jóvenes son:

Económicas: relacionadas con la ausencia de dinero en el hogar y con la necesidad de insertarse al mercado laboral.

Institucionales o escolares: se dividen en dos grupos: el de las intersistémicas, que aluden a "la oferta educativa, la desigualdad en la calidad de los servicios educativos y los mecanismos de acceso, asociados a la asignación de plantel, modalidad y turno..." (INEE, 2017a, p. 196), y el de las razones intrasistémicas que impactan en el bajo desempeño académico debido a factores asociados a "prácticas pedagógicas inadecuadas, formación docente limitada y condiciones laborales precarias, infraestructura y equipamiento insuficiente, incompatibilidad entre la cultura juvenil y escolar, currículo poco pertinente, gestión escolar deficiente, y participación limitada de padres y estudiantes en la escuela" (INEE, 2017a, p. 196).

Familiares e individuales: para Székely (2015) y Weiss (2015), en las primeras se ubica la escolaridad de los padres y el hecho de vivir, o no, con ambos; en las segundas están la desmotivación, el desinterés por la escuela y problemas propios de la identidad juvenil, como las adicciones, la violencia o el embarazo a edad temprana.

Al respecto de los tres grupos de razones anteriores, pueden subrayarse los datos y hallazgos más relevantes. Los resultados de la Encuesta Nacional de Ocupación y Empleo (Instituto Nacional de Estadística y Geografía [INEGI], 2009) señalan que $52 \%$ de las causas de abandono se asocian a la falta de dinero para pagar la escuela y la necesidad de aportar económicamente al hogar (Subsecretaría de Educación Media Superior y Consejo para la Evaluación de la Educación del Tipo Medio Superior, citados en INEE, 2017b).

El reporte de la Encuesta Nacional de Deserción en la Educación Media Superior (SEP y Consejo para la Evaluación de la Educación del Tipo Medio Superior, 2012) señala que más de una tercera parte de los jóvenes (35.4\%) abandonan la escuela por causas económicas; asimismo, indica que alrededor de una tercera parte de los jóvenes (32.3\%) dejan la escuela por causas escolares-institucionales.

En los resultados del "Análisis del movimiento contra el abandono escolar en la EMS" (SEP e Instituto Nacional de Salud Pública, 2015) destaca que 38.0\% de los jóvenes abandonan la escuela por razones eminentemente económicas. Este estudio coloca las causas escolares-institucionales como las más importantes, debido a que $41.0 \%$ de los estudiantes las señalan como la razón fundamental para dejar sus estudios. 
En otras líneas de análisis se ha mencionado que las condiciones de riesgo social, como la exclusión y la polarización, así como las dificultades para construir identidad, explican también una parte importante del abandono de los jóvenes en la EMS (Miranda, 2012b y 2013); esto es, la segmentación social se configura en un rasgo característico de los jóvenes.

Basta con citar algunos datos para ilustrarlo. Con base en la Encuesta Intercensal de 2015 (INEGI, 2015), la población en este grupo de edad ascendía en ese año a 6.4 millones, lo que representaba $5.4 \%$ respecto de la población total. La mitad eran hombres y la mitad, mujeres (50.4 y 49.6\%, respectivamente). Si bien $23.0 \%$ se consideraba indígena en cierta medida, solo $6.1 \%$ hablaba alguna lengua indígena; $75.0 \%$ habitaba en zonas urbanas, pero una cuarta parte vivía en localidades con menos de 2,500 habitantes (rurales) (INEGI, 2015).

Para estos jóvenes, la incidencia de la pobreza es preocupante. De acuerdo con los datos sobre pobreza y carencia social del Consejo Nacional de Evaluación de la Política de Desarrollo Social (Coneval, 2017), mientras 39.9\% de la población mexicana de 18 años y más vive en condiciones de pobreza multidimensional, el grupo de edad de 15 a 17 años en esta misma condición es de 48.1\%. De forma similar, la cantidad de jóvenes en pobreza extrema también es mayor (7.8\% en contraste con 6.9 de población mayor de 18 años), así como los que presentan carencia social de acceso a seguridad social (64.3\% en comparación con 54.3), alimentación (24.3\% frente a 18.7), vivienda (14.2\% respecto a 10.2$)$ e ingresos (17.7\% de la población de 15 a 17 años tiene ingresos menores que la línea de bienestar en comparación con $15.5 \%$ de la población mayor de 18 años).

Estas cifras permiten concluir que esta población, dadas sus condiciones de segmentación social, no asiste a la escuela o, en el mejor de los casos, aun asistiendo, se encuentran en riesgo de abandonar su educación o ya lo han hecho. A esto se suman cuestiones específicas de identidad de los jóvenes, quienes, como se ha señalado en estudios previos sobre las tensiones entre las culturas juveniles y la cultura escolar,

cuentan con múltiples formas de expresión cultural, ideológica y política; con diferentes expectativas de su proyecto de vida; con maneras variadas del uso del cuerpo: en ellos permean el hedonismo y los procesos de construcción de intimidad, las manifestaciones de sexualidad y las distintas formas de socialización alternativa que las culturas institucionalizadas como las de la escuela tienden a rechazar (Bracho y Miranda, 2017, p. 17).

En suma, las causas son múltiples, variadas y cada una tiene distinta naturaleza, pero todas se interrelacionan y configuran un complejo entramado de factores que deberán ser solventados según las situaciones y características específicas de las que se trate.

\section{APORTES TEÓRICOS Y EXPERIENCIAS INTERNACIONALES}

\section{Aportes teóricos: hacia un marco analítico del abandono escolar}

Aun cuando se ha avanzado en los estudios empíricos sobre las causas del abandono escolar, es poco lo que se ha discutido teóricamente sobre el tema. Si bien no existe una teoría del abandono escolar de los jóvenes, es posible avanzar algunos aportes en torno a tres enfoques analíticos que se consideran pertinentes 
para integrar y analizar las diversas y múltiples causas del abandono escolar. Tales aportes se alinean con la noción de riesgo social, procesos de des-subjetivación de los jóvenes y desafiliación escolar que experimentan. A continuación describimos de manera breve cada uno de ellos:

\section{Riesgo social}

Es la exposición de los adolescentes y jóvenes a circunstancias que incrementan sus probabilidades de sufrir daño en su integridad física, psicosocial, moral o social con el efecto consecuente en la disminución de sus posibilidades de educabilidad, es decir, se ven restringidas sus oportunidades para acceder a una educación digna, suficiente y de buena calidad (Hammond, Linton, Smink \& Drew, 2007).

Dicha exposición atiende, en primer lugar, el hecho de que la condición juvenil por sí misma plantea situaciones generalizadas de riesgo; esto es, ser joven es una "clase de edad" en la que se es más sensible a diversos problemas: definición de identidad asociada a los procesos de maduración psicológica y social; violencia; consumo de drogas; redescubrimiento de la sexualidad; búsqueda de la independencia económica; y diversos aspectos de la condición ideológica, moral o religiosa (Organización de las Naciones Unidas para la Educación, la Ciencia y la Cultura [Unesco], 2017; Fondo Internacional de Emergencia de las Naciones Unidas para la Infancia [Unicef], 2016 y 2017).

En segundo lugar, responde a los problemas fundamentales de equidad, bienestar y desarrollo social; con ello los riesgos de "ser joven" se multiplican cuando tienen que enfrentar otras condiciones de vulnerabilidad (exclusión, pobreza o carencia) asociadas a su situación socioeconómica, geográfica, étnica o biológica (Montgomery \& Hewett, 2005; Ruel, Haddad \& Garrett, 2008; Unicef, 2016 y 2017).

A lo anterior se suma la diversidad de las "culturas juveniles" que se expresan en diversos contextos geográficos y socioeconómicos a través de ciertas prácticas concretas, formas de consumo cultural y expresiones antisistémicas (Mockus, 2010; Reguillo, 2000).

En resumen, en la perspectiva del riesgo social, los adolescentes y jóvenes se encuentran altamente diferenciados tanto en su existencia material como simbólica, en diferentes contextos socioeconómicos y geográficos (rural, urbano, ciudades pequeñas, medianas y megalópolis), caracterizados por la heterogeneidad estructural, la diversidad cultural e ideológica y, sobre todo, la inequidad de oportunidades sociales y educativas.

\section{Des-subjetivación}

Este enfoque se nutre de las aportaciones teóricas provenientes de la sociología de la experiencia escolar planteada por Dubet y Martuccelli (1998). Ambos centran su investigación en la importancia que tiene el entorno escolar en la permanencia de los estudiantes. Conciben la escuela como "un aparato reproductor" que no solo produce calificaciones y competencias, sino también individuos con actividades y disposiciones. De acuerdo con esta perspectiva, la escuela reproduce condiciones desiguales que promueven el abandono escolar. Así, la experiencia escolar es producto del ambiente 
escolar, el cual tiene un efecto distinto entre los estudiantes, ya que puede ser un marco "de socialización y construcción de ciudadanía para unos y [...] un obstáculo para otros" (Miranda, 2012b, p. 6).

Dubet y Martuccelli (1996) afirman que "en cuanto más débiles son los alumnos, menos capacidades de socialización posee la escuela y más se constituye la vida juvenil al margen de la escuela o en contra de ella. En los medios favorecidos esta tendencia es la más acusada" (p. 453). Así, la construcción o el fortalecimiento de la subjetividad de los jóvenes en la escuela se dificulta, debido a que la diversidad juvenil -por cuanto hace a sus crecientes diferencias en sus capitales sociales y culturales- enfrenta con mayores problemas la tensión entre la "lógica estratégica" y la "lógica de integración" en contextos donde la competencia y la selección se expresan con mayor dureza frente a las presiones del mercado, del prestigio de las profesiones y del futuro social.

En este marco reconocemos, por un lado, que los estudiantes atendidos en la actualidad no solo son más, sino que son diferentes cultural y morfológicamente, y por el otro, que la actual y tradicional oferta educativa está sumergida en un contexto caracterizado por "el derrumbe de las antiguas formas pedagógicas, el debilitamiento del prestigio de los docentes, la competencia de la cultura escolar y de cultura de masas más seductoras y más poderosas, así como a la llegada de nuevos alumnos 'inadaptados'” (Dubet y Martuccelli, 1998, p. 56).

Esta tensión que no se resuelve para muchos jóvenes produce la salida del aparato escolar, o bien, una permanencia instrumentalista con el consecuente déficit de identidad, subjetividad y autonomía en el proyecto social e individual de los jóvenes (Filmus y Moragues, 2003; Morduchowicz, 2003; Quevedo, 2003; Tenti, 2003).

Desafiliación escolar (desintegración académica y social)

Vincent Tinto (1975 y 1989) distingue entre "exclusión académica" y "deserción voluntaria". Sin embargo, considera que la mayoría de los procesos de abandono escolar son voluntarios y se dan, con mayor frecuencia, en los primeros meses posteriores al ingreso. Su origen se ubica en la insuficiente "integración personal" del estudiante con el "ambiente intelectual y social" de la escuela-institución (Tinto, 1975). Subraya que, si bien el abandono escolar es el resultado de una decisión individual, esta no solo depende de las características individuales, sino también es consecuencia del ambiente institucional, de procesos sociales e intelectuales, en particular resultado de la ausencia de integración del estudiante con su institución (Tinto, 1989). Así, este fenómeno no se da de manera espontánea, sino por un proceso de desgaste del estudiante (student attrition) (Tinto, 1982).

Con base en el "Modelo de la persistencia", Tinto (1975) afirma que la decisión de permanecer en los estudios es resultado del ajuste que hay entre el estudiante y la institución, lo que genera integración académica y social entre ambos. Por tanto, la participación en distintos grupos dentro de la comunidad, el trabajo colaborativo y el acompañamiento favorecen la integración y generan la persistencia en los estudios. En contraste, el abandono escolar es visto como el fracaso de los individuos para integrarse a una comunidad educativa (Tinto, 1975). 
Como se ha dicho en otros estudios, esta perspectiva permite afirmar, desde el punto de vista individual, que "los jóvenes que abandonan la escuela, aquellos que se desafilian, son precisamente quienes no lograron construir un sentido de comunidad y de identificación mínima con la institución" (Miranda, 2012b, p. 78).

Desde la lógica de la escuela-institución, el abandono escolar es resultado de la incapacidad de esta y de la política pública para contener a los estudiantes que, por sus condiciones socioculturales, se encuentran más alejados de las exigencias escolares. Por ello, habría que crear los mecanismos adecuados para que no se "desafilien" y logren concluir su trayectoria escolar.

En síntesis, si bien es cierto que varios de los problemas asociados al abandono son externos a la escuela, no lo es menos que esta los agudiza y se ven reflejados en rezago, des-subjetivación, desafiliación y abandono escolar. Por ello, este enfoque propone la redefinición del papel de la escuela en el proceso de enseñanza-aprendizaje y en la permanencia escolar de los estudiantes, vista como un espacio incluyente y potenciador de una educación que no solo integre individuos a la sociedad, sino que también sea formadora de sujetos en igualdad de condiciones.

El marco analítico presentado no considera que alguna de las dimensiones mencionadas (riesgo social, des-subjetivación y desafiliación) articulen factores que sean inamovibles ni tampoco del todo exógenos a la escuela. Por tanto, mediante acciones concretas en cada una de estas dimensiones se puede lograr un adecuado combate al abandono escolar.

La figura sintetiza las categorías problema del abandono escolar y para cada una de ellas se explica la acción pública requerida para enfrentarlas con un esquema articulado de intervenciones sistemáticas; es decir, la acción pública debe dirigirse no solo a la contención social, la cual solo considera algunos elementos de la dimensión de riesgo social, sino que debe implicar acciones que incentiven procesos de integración académica y social de los jóvenes para atacar la desafiliación escolar, así como de resignificación de la experiencia escolar para fortalecer la identidad de los sujetos y sus proyectos sociales e individuales de vida.

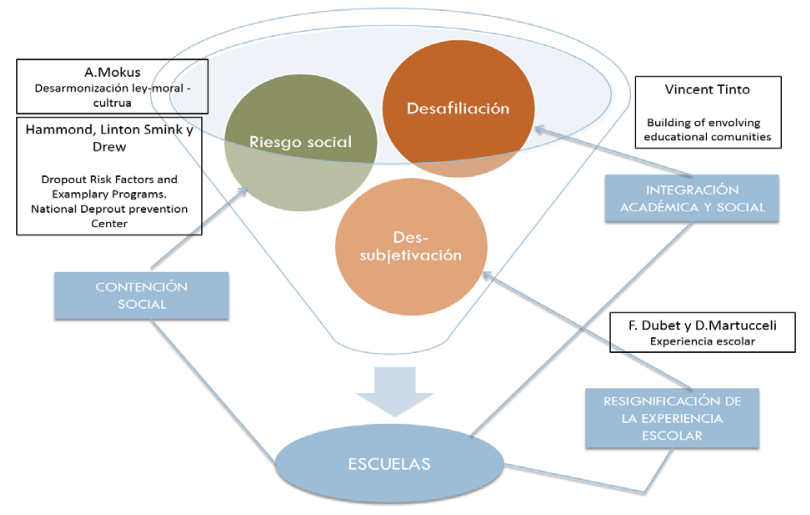

Figura. Esquema analítico del abandono escolar y la acción pública para su combate. Fuente: elaboración propia con base en los autores consultados. 
El abandono escolar se presenta como un problema complejo que responde de manera simultánea y con diversos grados de intensidad a factores económicos, institucionales o escolares, familiares e individuales. Por ello, heurísticamente, no es adecuado relacionar "uno a uno" a los factores que causan el abandono escolar con los elementos teóricos establecidos. Sin embargo, con la finalidad de elaborar un mapa analítico que permita avanzar en la caracterización de las intervenciones gubernamentales en la materia, a partir de los elementos teóricos señalados, buscamos ordenar los ámbitos o ejes de intervención pública para atender el abandono escolar considerando el énfasis que reflejan respecto a los factores o causas principales que lo generan.

De este modo, pretendemos precisar, con base en diferentes intervenciones públicas gubernamentales, la manera de enfrentar el riesgo que produce la insuficiencia económica o material y que afecta la equidad en las oportunidades sociales y educativas de importantes grupos de adolescentes y jóvenes. En un sentido similar, buscamos dar cuenta de aquellas acciones o programas orientados a mitigar los riesgos familiares o individuales, así como los procesos de segmentación producidos al interior del sistema y en los propios planteles escolares y que crean problemas importantes de "construcción de sentido" para enganchar a los jóvenes con la cultura académica, fortalecer su identidad y apoyar su permanencia escolar.

A continuación mostramos algunas experiencias internacionales exitosas sobre el tema con la finalidad de tenerlas presentes en el análisis para atender el abandono escolar en la EMS.

\section{Algunas experiencias internacionales}

Los siguientes son aportes y experiencias internacionales relevantes que se orientan a la mejora de la permanencia en la escuela:

\section{National Dropout Prevention Center}

Es un espacio que comparte información sobre la prevención del abandono escolar y ofrece estrategias efectivas para aumentar las tasas de graduación. Es un recurso nacional en Estados Unidos cuyo propósito es brindar soluciones para garantizar la permanencia y el éxito de los estudiantes a través del intercambio de información, proyectos de investigación y evaluación, publicaciones y diversas actividades de desarrollo profesional. Además, lleva a cabo revisiones y evaluaciones de los programas.

Este centro utiliza como eje de sus análisis intervenciones institucionales y estrategias de movilización social para enfrentar los problemas de deserción escolar que afectan a adolescentes y jóvenes, y un conjunto de predictores de riesgo de abandono escolar agrupados en cuatro dimensiones: relacionados con la escuela; relacionados con la comunidad; relacionados con el estudiante; y relacionados con la familia.

\section{Graduate XXI \& Futuro Educativo}

Es un proyecto en el que trabajan seis investigadores y especialistas que laboran en la división de Relaciones Externas del Banco Interamericano de Desarrollo, el cual 
cuenta con el apoyo de la República de Corea del Sur. Su objetivo es que surjan nuevas soluciones educativas innovadoras que puedan generar un efecto multiplicador y transformador para la permanencia escolar de los estudiantes. Entre sus principales acciones, destaca el aprovechamiento de las oportunidades que ofrecen las nuevas tecnologías, mediante el desarrollo de herramientas tecnológicas que favorezcan la conectividad para crear espacios virtuales en los que se propicie el debate público y se promueva la concientización de la sociedad sobre esta problemática en la región. Todo ello tiene la finalidad de contribuir a prevenir y disminuir los índices de abandono escolar.

\section{Estrategia 2020 y Kit de Herramientas Europeas para Centros Escolares}

La estrategia fue establecida en 2010 y plantea cinco objetivos, de los cuales destaca uno orientado a la política educativa: "Reducir la tasa de abandono escolar a menos del $10.0 \%$ y aumentar hasta al menos el $40.0 \%$ la tasa de titulados de la enseñanza superior" (Unión Europea, s.f., párrafo 11). Este objetivo se centró en dos niveles educativos: el nivel medio superior y el superior. Respecto al objetivo de la Estrategia Europea 2020, dirigido a la educación media superior, observamos la necesidad de reducir el abandono escolar prematuro, cuya definición misma reconoce la importancia de la formación de los jóvenes en la EMS como una parte fundamental para la sociedad europea, su crecimiento y desarrollo económico, así como una manera de mejorar las capacidades para enfrentarse a factores de riesgo social complejos, como el desempleo y la pobreza (Comisión Europea, 2011, p. 2).

Experiencias como las anteriores son elementos de referencia importantes para analizar las políticas e intervenciones gubernamentales desarrolladas en México.

\section{ACCIONES DE POLÍtica PÚBLICA EN MÉXICO Y LAS DIRECTRICES EMITIDAS POR EL INEE}

El análisis de las acciones principales de política pública desarrolladas en el país para atender el problema de abandono en la EMS tiene la finalidad de hacer un balance más ordenado y analítico de ellas, a partir del marco conceptual antes expuesto.

\section{Mapeo de política pública}

Con base en el mapeo de la acción pública realizado por el INEE (2017a) y dirigido a la prevención y atención del abandono escolar en EMS, identificamos 16 acciones, estrategias y programas (ver tabla 1) orientados a ofrecer distintos tipos de apoyos: económicos (nueve programas), desarrollo socioemocional (tres programas) y académicos (cuatro programas).

Uno de los apoyos económicos para la permanencia en la EMS es el Programa Nacional de Becas (PNB) y el Programa Prospera, Programa de Inclusión Social. En cuanto a los apoyos socioemocionales, destaca el Programa Construye T. En tanto que el Movimiento contra el Abandono Escolar, Yo no abandono, recupera las intervenciones como los instrumentos de identificación de los alumnos en riesgo de abandono, el programa de tutorías académicas y el de orientación vocacional (INEE, 2017b). 
Respecto al presupuesto asignado, con base en el análisis del gasto federal dirigido a atender el abandono escolar en EMS (INEE, 2017b), se concluye que los estímulos económicos que brinda Prospera y los apoyos del PNB son las acciones que mayor porcentaje destinan para el abatimiento del abandono escolar $(93.2 \%)$. Mientras tanto, solo 6.8\% de los recursos se dirigen a la atención de factores de otra naturaleza (Secretaría de Hacienda y Crédito Público, 2017, citado en INEE, 2017b).

En un esfuerzo de sistematización de los principales resultados de las evaluaciones a cuatro programas que integran la actual política de atención al abandono escolar en EMS, pueden mencionarse los siguientes hallazgos:

- Los resultados de las evaluaciones del PNB y Prospera indican la necesidad de robustecer la visión general del problema del abandono escolar a nivel nacional, esclarecer la población objetivo, así como sustentar de qué manera las becas tienen un impacto en aumentar las tasas de transición de un nivel a otro (Centro de Investigación y Docencia Económicas y Centro para el Aprendizaje en Evaluación y Resultados, 2016; Estrategias de Acompañamiento y Servicios Educativos, 2015).

- Los principales hallazgos de la evaluación del Programa Construye T revelan que el programa ha modificado las prácticas y concepciones de los docentes. Sin embargo, los resultados también señalan la necesidad de potenciar las capacidades de la escuela para desarrollar las habilidades socioemocionales de los jóvenes y mejorar el ambiente escolar (IIPE-Unesco, 2010).

-Es necesario fortalecer el Movimiento contra el Abandono Escolar, debido a que los resultados de su evaluación exponen la falta de elementos robustos de formación, orientación y guía de los tutores. Asimismo, se manifiesta una falta de reconocimiento de las grandes diferencias entre subsistemas, modalidades y planteles por tipo de sostenimiento (UNAM, 2016; Valora, 2016).

A partir de los resultados de estas evaluaciones, se subraya, por un lado, que la intencionalidad de las acciones de política tiende a diluirse ante el escaso acompañamiento y seguimiento en la implementación de las propuestas de intervención, además de las dificultades de los planteles para ejecutar las acciones por sí solos. Por otro, se destaca la necesidad de fortalecer el liderazgo de los actores escolares a fin de identificar los riesgos que enfrentan los jóvenes para tener trayectorias escolares exitosas, sin que ello represente cargas extras de trabajo, así como impulsar procesos de seguimiento y evaluación, y reforzar los mecanismos de transparencia y rendición de cuentas.

Para ofrecer un balance más analítico de estos programas, la tabla 1 presenta un cruce de estos con las categorías analíticas consideradas en el esquema teórico señalado en este trabajo. En términos generales, la acción gubernamental ha puesto mayor hincapié en la contención del riesgo social y mucho menos en atender los problemas de des-subjetivación y desafiliación escolar. Parece que la política pública se ha centrado más en la atención de las causas económicas, y no en las institucionales o escolares, y menos aún en las familiares e individuales, aunque ya existen primeras iniciativas al respecto.

Destaca el hecho de que solo la estrategia de Construye T consideró la dimensión de des-subjetivación en su primera etapa (pero no en la actual). Por otro lado, si bien 
el programa Síguele considera una instancia de la cultura juvenil, con el programa de fomento a la lectura, no ha tomado en cuenta medios de adaptación a la cultura de los jóvenes. En suma, los esfuerzos en torno a esta cuestión han sido limitados y no han considerado a los jóvenes para su construcción.

Tabla 1. Programas, acciones y estrategias que atienden el abandono escolar en educación media superior, según dimensión teórico-conceptual

\begin{tabular}{|c|c|c|c|c|c|c|c|}
\hline \multirow{3}{*}{$\begin{array}{c}\text { Programas, acciones y } \\
\text { estrategias }\end{array}$} & \multicolumn{7}{|c|}{ Dimensión } \\
\hline & \multicolumn{2}{|c|}{ Riesgo social } & \multicolumn{3}{|c|}{ Desafiliación } & \multicolumn{2}{|c|}{ Des-subjetivación } \\
\hline & $\begin{array}{c}\text { Factores de } \\
\text { riesgo material }\end{array}$ & $\begin{array}{l}\text { Cultura } \\
\text { juvenil }\end{array}$ & $\begin{array}{l}\text { Partici- } \\
\text { pación }\end{array}$ & $\begin{array}{c}\text { Trabajo } \\
\text { colaborativo }\end{array}$ & $\begin{array}{l}\text { Acompaña- } \\
\text { miento }\end{array}$ & $\begin{array}{l}\text { Ambiente } \\
\text { escolar }\end{array}$ & Integración \\
\hline $\begin{array}{l}\text { 1. Programa Nacional } \\
\text { de Becas de Excelencia } \\
\text { Académica en EMS }\end{array}$ & $\mathrm{X}$ & --- & --- & --- & --- & --- & --- \\
\hline $\begin{array}{l}\text { 2. Programa Nacional } \\
\text { de Becas de Apoyo a } \\
\text { Estudiantes en Educa- } \\
\text { ción Media Superior }\end{array}$ & $\mathrm{X}$ & --- & --- & --- & --- & --- & --- \\
\hline $\begin{array}{l}\text { 3. Programa Nacional } \\
\text { de Becas para la Re- } \\
\text { tención de Estudiantes } \\
\text { en Educación Media } \\
\text { Superior }\end{array}$ & $\mathrm{X}$ & --- & --- & --- & --- & --- & --- \\
\hline $\begin{array}{c}\text { 4. Programa de } \\
\text { Atención Educativa a } \\
\text { Grupos en Situación } \\
\text { Vulnerable }\end{array}$ & $\mathrm{X}$ & --- & --- & --- & --- & --- & --- \\
\hline $\begin{array}{l}\text { 5. Programa Becas } \\
\text { de Educación Media } \\
\text { Superior }\end{array}$ & $\mathrm{X}$ & --- & --- & --- & --- & --- & --- \\
\hline $\begin{array}{l}\text { 6. Programa de Becas } \\
\text { para la Expansión de } \\
\text { la Educación Media } \\
\text { Superior, Síguele }\end{array}$ & $\mathrm{X}$ & --- & --- & --- & --- & --- & --- \\
\hline $\begin{array}{l}\text { 7. Programa de Becas } \\
\text { para Acceder, Per- } \\
\text { manecer y Concluir } \\
\text { la Educación Media } \\
\text { Superior }\end{array}$ & $\mathrm{X}$ & --- & --- & --- & --- & --- & --- \\
\hline $\begin{array}{l}\text { 8. Programa Nacional } \\
\text { de Becas }\end{array}$ & $\mathrm{X}$ & --- & --- & --- & --- & --- & --- \\
\hline $\begin{array}{l}\text { 9. Programa de Inclu- } \\
\text { sión Social, Prospera }\end{array}$ & $\mathrm{X}$ & --- & --- & --- & --- & --- & --- \\
\hline $\begin{array}{l}\text { 10. Programa } \\
\text { Construye T }\end{array}$ & & $\mathrm{X}$ & $\mathrm{X}$ & & $\mathrm{X}$ & $\mathrm{X}$ & $\mathrm{X}$ \\
\hline $\begin{array}{l}\text { 11. Programa Síguele, } \\
\text { caminemos juntos }\end{array}$ & $\mathrm{X}$ & --- & $\mathrm{X}$ & $\mathrm{X}$ & $\mathrm{X}$ & --- & --- \\
\hline $\begin{array}{l}\text { 12. Movimiento contra } \\
\text { el Abandono Escolar, } \\
\text { Yo no abandono }\end{array}$ & $\mathrm{X}$ & --- & $\mathrm{X}$ & $\mathrm{X}$ & $\mathrm{X}$ & --- & --- \\
\hline $\begin{array}{l}\text { 13. Sistema Nacional } \\
\text { de Tutorías Académi- } \\
\text { cas, Síguele }\end{array}$ & --- & --- & --- & --- & $\mathrm{X}$ & --- & --- \\
\hline $\begin{array}{c}\text { 14. Programa de } \\
\text { Orientación Vocacional, } \\
\text { Síguele }\end{array}$ & --- & --- & --- & --- & $X$ & --- & --- \\
\hline $\begin{array}{l}\text { 15. Sistema de Alerta } \\
\text { Temprana, Síguele }\end{array}$ & $X$ & --- & --- & --- & --- & --- & --- \\
\hline $\begin{array}{c}\text { 16. Programa de } \\
\text { Fomento a la Lectura, } \\
\text { Síguele }\end{array}$ & --- & --- & $X$ & --- & --- & --- & --- \\
\hline
\end{tabular}


Directrices para mejorar la permanencia escolar en la educación media superior

Estas directrices se fundamentan en diversas evaluaciones que desarrolla el INEE en EMS: Plan Nacional de Evaluación de los Aprendizajes y Evaluación de la Oferta Educativa, así como las evaluaciones de la política educativa de atención al abandono escolar en este nivel. Además, se tomaron en cuenta estudios coordinados por el propio INEE sobre el funcionamiento institucional y la oferta educativa en EMS. Destacan también diversos espacios de interlocución con autoridades educativas, tanto en el ámbito federal como local.

Con base en lo anterior, el INEE emitió en 2017 las Directrices para mejorar la permanencia escolar en la educación media superior. Estas buscan consolidar políticas integrales que contribuyan a garantizar la permanencia escolar en la EMS; desarrollar y fortalecer opciones viables para que los estudiantes que interrumpen sus estudios tengan la posibilidad de terminar la EMS; y propiciar entornos de aprendizaje que motiven a los estudiantes a continuar y terminar la EMS (INEE, 2017a).

A partir de la evidencia reportada por la investigación y la evaluación educativa disponible, las directrices emitidas por el INEE buscan establecer bases normativas y operacionales para que en cada plantel escolar existan mecanismos de prevención y protocolos de atención inmediata a jóvenes que dejan de asistir a la escuela, así como dispositivos de reincorporación educativa para jóvenes con más tiempo de haber abandonado sus estudios. Para ello, se plantea un esquema general de política educativa que articule los distintos niveles de gobierno y, en especial, a todos los subsistemas y modalidades educativas para que se fortalezca la coordinación interinstitucional y, en consecuencia, la permanencia escolar de los jóvenes.

A la par, con estas directrices se insiste en colocar la agenda de la permanencia escolar en los diversos espacios colegiados, como el Consejo Nacional de Autoridades Educativas (2017) y, en el ámbito local, reactivar los Comités Estatales para la Planeación y Programación de la Educación Media Superior.

En esta perspectiva de coordinación interinstitucional y de trabajo colegiado, en las directrices se reitera discutir temas clave como los vinculados a los esfuerzos de flexibilización curricular, incrementos presupuestarios, atención a los déficits acumulados de aprendizaje con los que ingresan los alumnos a la educación media superior, y la revisión de la gestión de becas y su impacto en la retención escolar.

Además, las directrices desglosan otras recomendaciones de política que apuntan a componentes afirmativos trascendentes, como la relativa al fortalecimiento de los tutores académicos a partir de estrategias de profesionalización y la garantía de condiciones institucionales para su adecuado desempeño. Un factor central de la propuesta formulada por el INEE radica en la incorporación del tema de la permanencia escolar en el centro de la formación y las prácticas docentes a efecto de garantizar interacciones pedagógicas pertinentes a las necesidades educativas de los jóvenes.

Se añaden a lo anterior dos temas estratégicos: por una parte, la reconstrucción de la identidad de los jóvenes con la escuela a partir de esquemas de fortalecimiento de la seguridad, la inclusión educativa y social, y la participación democrática, pensados todos en la lógica del afianzamiento y reapropiación de la institucionalidad 
escolar; y por otra, la ampliación de las oportunidades de reincorporación educativa para jóvenes que llevan mucho tiempo de haberse desprendido de la escuela buscando recuperar su mundo de vida personal, familiar y laboral en estrategias pertinentes de culminación de sus estudios.

En la tabla 2 incluimos la denominación de cada una de las directrices, así como el número de aspectos clave de mejora que cada una incorpora.

Tabla 2. Directrices y aspectos clave de mejora

\begin{tabular}{|l|c|}
\hline \multicolumn{1}{|c|}{ Directriz } & $\begin{array}{c}\text { Número de aspectos } \\
\text { clave de mejora }\end{array}$ \\
\hline $\begin{array}{l}\text { Fortalecer, con un enfoque de equidad, las políticas dirigidas a institucionalizar las accio- } \\
\text { nes para la permanencia escolar en los planteles de EMS }\end{array}$ & 10 \\
\hline $\begin{array}{l}\text { Mejorar la formación de los tutores académicos y las condiciones institucionales para su } \\
\text { adecuado desempeño }\end{array}$ & 8 \\
\hline $\begin{array}{l}\text { Fortalecer las competencias docentes para generar interacciones pedagógicas pertinen- } \\
\text { tes a las necesidades educativas de los jóvenes }\end{array}$ & 5 \\
\hline $\begin{array}{l}\text { Afianzar la identidad de los jóvenes con la escuela y promover ambientes escolares segu- } \\
\text { ros, incluyentes y democráticos }\end{array}$ & 7 \\
\hline $\begin{array}{l}\text { Ampliar las estrategias de reincorporación educativa de los jóvenes y atender la diversi- } \\
\text { dad de sus contextos sociales }\end{array}$ & 6 \\
\hline \multicolumn{1}{|c|}{ Total } & 36 \\
\hline
\end{tabular}

Fuente: elaboración propia con base en INEE, 2017a.

Desde una visión más analítica, fundamentada en los conceptos planteados en el apartado teórico de este trabajo (ver tabla 3), las dimensiones más atendidas por las directrices son el riesgo social y la desafiliación. La primera se recupera en las directrices 1,4 y 5 , en las que destaca la atención a un tema muy poco abordado por las políticas públicas, como lo es la cultura juvenil. Las directrices 2 y 3 se concentran en la dimensión de desafiliación con base en el fortalecimiento de capacidades y habilidades de docentes y tutores académicos; esto, con la finalidad de generar procesos de seguimiento y acompañamiento para incentivar la integración académica y social.

Por último, la des-subjetivación es retomada sobre todo en las directrices 1 y 4 , con el propósito de desarrollar estrategias que atiendan esta dimensión, considerada como la menos atendida por la acción pública. Además, las directrices que las prevén (1 y 4) están acompañadas por elementos que son incluidos en la dimensión de riesgo social (ver tabla 3).

Tabla 3. Cruce de directrices con dimensiones del marco analítico

\begin{tabular}{|c|c|c|c|c|c|c|}
\hline \multirow{2}{*}{ Dimensiones } & Categorías & Directriz & Directriz & Directriz & Directriz & Directriz \\
& 1 & 2 & 3 & 4 & 5 \\
\hline \multirow{3}{*}{ Riesgo social } & $\begin{array}{c}\text { Factores de riesgo } \\
\text { material }\end{array}$ & $\mathrm{X}$ & --- & --- & $\mathrm{X}$ & $\mathrm{X}$ \\
\cline { 2 - 7 } & Cultura juvenil & $\mathrm{X}$ & --- & --- & $\mathrm{X}$ & --- \\
\hline \multirow{2}{*}{ Des-subjetivación } & Ambiente escolar & --- & --- & --- & $\mathrm{X}$ & --- \\
\cline { 2 - 7 } & Integración & $\mathrm{X}$ & --- & --- & $\mathrm{X}$ & --- \\
\hline \multirow{2}{*}{ Desafiliación } & Participación & $\mathrm{X}$ & --- & --- & $\mathrm{X}$ & --- \\
\cline { 2 - 7 } & Trabajo colaborativo & --- & $\mathrm{X}$ & $\mathrm{X}$ & --- & --- \\
\cline { 2 - 7 } & Acompanamiento & $\mathrm{X}$ & $\mathrm{X}$ & $\mathrm{X}$ & --- & $\mathrm{X}$ \\
\hline
\end{tabular}


En términos generales, con base en el análisis de las acciones, estrategias y programas orientados a resolver el problema del abandono escolar en la EMS, es posible afirmar que las acciones de política pública, además de dirigirse a atender y garantizar condiciones mínimas de permanencia, deberán tomar en cuenta para su diseño los factores causales escolares y extraescolares relacionados con la diversidad social y cultural de los jóvenes, así como la falta de pertinencia y relevancia de las experiencias escolares en la EMS.

\section{A MANERA DE CONCLUSión: AVANCES Y RETOS DE LA POLÍtica PÚBLICA}

El cumplimento del derecho a una educación de calidad para todos, como lo establece la Constitución Política de los Estados Unidos Mexicanos, al igual que muchas constituciones de la mayoría de los países iberoamericanos, implica necesariamente cumplir con el derecho de acceso a la educación y, de manera puntual, el derecho a permanecer en la escuela y, por supuesto, a adquirir conocimientos relevantes.

El acceso, la permanencia y el logro educativo se inscriben en una secuencia de efectos de interacción relevantes: la calidad en los aprendizajes de los educandos, como fin último de la educación, depende en alta medida de las condiciones del acceso, la pertinencia y calidad de los servicios educativos (ubicación geográfica de la escuela, infraestructura, equipamiento, docentes, materiales educativos y procesos de gestión, entre otros), además de la pertinencia, equidad y calidad de las oportunidades de aprendizaje que se ofrecen a los educandos en función de sus capacidades y necesidades educativas. A ello se suma el importante papel que juegan las condiciones sociales de los educandos en las oportunidades educativas, tanto para potenciar como para restringir lo que la escuela ofrece.

La secuencia del derecho a la educación adquiere dimensiones críticas en la "clase de edad juvenil" por los diferentes procesos y condiciones que la caracterizan en las sociedades en desarrollo como las nuestras. Como lo hemos señalado en este artículo, esta "clase de edad" no solo está expuesta a condiciones de riesgo social, sino que su diversidad social y cultural, que es al mismo tiempo convergente y divergente con los códigos de la globalización, la posmodernidad y la pobreza, produce frente a la escuela y su oferta educativa serios procesos de desintegración social para una gran mayoría de jóvenes, además del efecto asociado de des-subjetivación y, en especial, de desafiliación educativa.

En esta perspectiva, el abandono escolar no es más que el efecto agregado de esos factores que cuestionan y clausuran una adecuada experiencia escolar para los jóvenes. De ahí que los predictores tradicionales de desafiliación escolar como el "no asiste", "no aprueba" y "no se comporta" deben trascender la fase de diagnóstico y avanzar hacia las de intervención educativa que no solo respondan a la necesidad de contención social de los riesgos, sino que replanteen los modelos educativos y escolares para hacerlos más flexibles y pertinentes a las necesidades y capacidades de los jóvenes. Por lo tanto, junto a la mejora de las capacidades institucionales de prevención de riesgos de abandono, es necesario involucrar más a las comunidades escolares para resignificar la experiencia escolar. Asimismo, además de garantizar condiciones mínimas de estancia (seguridad, atención, acceso), se requiere fortalecer la identidad de los jóvenes con la escuela. 
Tal condición y complejidad sociocultural del problema del abandono escolar de los jóvenes, como lo demuestra la teoría y la experiencia internacional comparada, requiere un enfoque integral de política pública en el cual lo educativo interactúe y dialogue con otros ámbitos sectoriales que permitan enfrentar y atender con mayor eficacia los problemas que se acentúan en la escuela, como la drogadicción, el embarazo temprano, la ciudadanía de baja intensidad, la inseguridad, la violencia y los diferentes procesos de "anomia social", entre otros.

Del mismo modo, es necesario reconocer el papel e importancia de los factores escolares como causales de abandono. A la precariedad de sus condiciones materiales y simbólicas, la inequidad social y la diversidad cultural de los jóvenes se suma la falta de pertinencia y de acciones afirmativas de orden pedagógico y social en la escuela. Sin duda, hacer frente a esta condición requiere replantear y movilizar en diversos sentidos alternativos los recursos educativos y escolares cobijados por una nueva idea de comunidad.

La experiencia mexicana de las políticas para abatir el abandono escolar muestra que, si bien se avanza en el camino adecuado, también manda señales para llenar vacíos, fortalecer acciones y replantear intervenciones institucionales y pedagógicas. Las enseñanzas del debate, la investigación y la evaluación sobre el desarrollo de política pública en México para atender el abandono escolar son claras al identificar las necesidades de los jóvenes. No obstante, se requiere implementar acciones más adecuadas y pertinentes a la realidad de este sector. En este sentido, y con el propósito de contribuir tanto al diseño de nuevas políticas y programas como a la implementación de los ya existentes, a continuación presentamos los siguientes elementos a considerar:

Realizar un giro lingüístico: no aludir a deserción escolar ni a abandono, sino al fortalecimiento de la permanencia escolar, a efecto de dejar de culpabilizar a los jóvenes y generar una cultura más integral de compromiso y responsabilidad de las instituciones y de la sociedad para atender el problema.

Repensar el abandono escolar en la educación media superior: desde la perspectiva de trayectorias, considerando los procesos de escolarización previa y la atención a los "déficits educativos acumulados", así como las rutas futuras y oportunidades educativas en el ámbito de la educación superior o de salidas al mundo del trabajo.

Reorganizar el sistema: replantear el archipiélago de subsistemas, modalidades y servicios, y avanzar hacia un sistema mejor comunicado y coordinado para favorecer la movilidad estudiantil y avanzar hacia ofertas educativas menos estratificadas y más sólidas en su capacidad de atención educativa.

Generar política pública integral para los jóvenes: en la cual la educación sea bisagra de procesos de afirmación social, psicológica, económica y ciudadana para los jóvenes.

Garantizar el compromiso institucional para fortalecer la permanencia escolar: esto es, definir política educativa a partir de un afianzamiento institucional en los distintos niveles de gobierno -federal, estatal y municipal-, a fin de que, en cada plantel, con el abrigo de la comunidad escolar, se institucionalicen diversos dispositivos de prevención, atención oportuna y reincorporación educativa para los jóvenes. 
"Pedagogizar" de la permanencia escolar: lo que significa convertir el problema en un asunto vinculado a la capacidad de los docentes y de la escuela para generar interacciones pedagógicas significativas para los jóvenes.

Fortalecer las acciones afirmativas: que permitan atender necesidades educativas específicas con base en las acciones de tutoría y acompañamiento cada vez más profesionales, con capacidad niveladora, pero también de integración académica y psicosocial de los jóvenes.

Fortalecer otras capacidades de los jóvenes: tales como el fortalecimiento socioemocional y la capacidad de resiliencia.

Promover la resignificación de la experiencia escolar: a partir de nuevos juegos identitarios con la escuela, la vinculación con proyectos comunitarios y el afianzamiento de las redes de protección social de los jóvenes.

Generar nuevos diálogos y sistemas de interacción: con el entorno económico y social de los jóvenes que pasa por el reconocimiento de sus procesos de emancipación, lo cual atañe al trabajo, al ejercicio de la ciudadanía y la vida independiente.

Ampliar y flexibilizar la oferta educativa: que vaya de la mano con el devenir juvenil por la sociedad y, sobre todo, con sus posibilidades de reincorporación educativa en atención a su condición familiar, personal, social y económica.

\section{REFERENCIAS BIBLIOGRÁFICAS}

Abril, E., Román, R., Cubillas, M., y Moreno, I. (2008). ¿Deserción o autoexclusión? Un análisis de las causas de abandono escolar en estudiantes de educación media superior en Sonora, México. REDIE. Revista Electrónica de Investigación Educativa, vol. 10, núm. 1, pp. 1-16.

Attanasio, 0. \& Székely, M. (2003). The family in flux: Household decision-making in Latin America. Washington, DC: Inter-American Development Bank.

Banco Interamericano de Desarrollo (2012). Desconectados. Habilidades, educación y empleo en América Latina. Washington, EUA.

Bracho, T. y Miranda, F. (2017). Desencuentros entre los jóvenes y la escuela en América Latina. Gaceta de la Política Nacional de Evaluación Educativa en México, vol. 3, núm. 7, pp. 16-22. Recuperado de http://www.inee.edu.mx/ images/stories/2017/Gaceta7/G07-esp.pdf

Centro de Investigación y Docencia Económicas y Centro para el Aprendizaje en Evaluación y Resultados (2016). Evaluación de diseño. Programa Nacional de Becas. CONEVAL/SEP.

Comisión Económica para América Latina y el Caribe (2015). Panorama social de América Latina. Santiago de Chile: Naciones Unidas/CEPAL.

Comisión Europea (2011). Comunicación de la comisión al parlamento europeo, al consejo, al comité económico y social europeo y al comité de las regiones. Bruselas, Bélgica.

Consejo Nacional de Autoridades Educativas (2017). Presentación de la LVIII Reunión del Consejo Nacional de Autoridades Educativas. Capítulo Educación Media Superior. Dr. Rodolfo Tuirán. 
Consejo Nacional de Evaluación de la Política de Desarrollo Social (2017). Resultados de pobreza en México 2016 a nivel nacional y por entidades federativas. México.

Constitución Política de los Estados Unidos Mexicanos (1917). México.

Dander, M. (2017). Entre la diversidad y la fragmentación: sobre el origen y desarrollo de la educación media superior en México. Gaceta de la Política Nacional de Evaluación Educativa en México, vol. 3, núm. 7, pp. 81-84. Recuperado de http://www.inee.edu.mx/images/stories/2017/Gaceta7/G07-esp.pdf

Diario Oficial de la Federación (2012, 9 de febrero). Decreto por el que se declara reformado el párrafo primero; el inciso c) de la fracción II y la fracción V del artículo 3o., y la fracción I del artículo 31 de la Constitución Política de los Estados Unidos Mexicanos.

Diario Oficial de la Federación (2008, 26 de septiembre.). Acuerdo número 442 por el que se establece el Sistema Nacional de Bachillerato en un marco de diversidad,

Dubet, F. y Martuccelli, D. (1998). En la escuela. Sociología de la experiencia escolar. España: Losada.

Estrategias de Acompañamiento y Servicios Educativos (2015). Evaluación cualitativa del nuevo esquema de entrega de becas educativas de Prospera, Programa de Inclusión Social. Entregable 4: Informe final. México.

Filmus, D. y Moragues, M. (2003). ¿Para qué universalizar la educación media? En E. Tenti (ed.). Educación media para todos. Los desafíos de la democratización del acceso (pp. 36-64). Argentina: Unesco/IIPE/Altamira/OSDE.

Fondo de las Naciones Unidas para la infancia (Unicef) (2016). Una exploración sobre la situación de las y los adolescentes y jóvenes en América Latina y el Caribe a partir de evidencia cuantitativa reciente. Nueva York.

Fondo de las Naciones Unidas para la Infancia (Unicef) (2017). Estado mundial de la infancia 2017. Nueva York.

Gobierno de la República (2013). Plan Nacional de Desarrollo 2013-2018. México.

Goicovic, I. (2002). Educación, deserción escolar e integración laboral juvenil. Última Década, núm. 16, pp. 11-53.

Hammond, C., Linton, D., Smink, J. \& Drew, S. (2007). Dropout risk factors and exemplary programs. A technical report. National Dropout Prevention Center/Network-Communities In Schools, Inc.

Huerta, R. (2010). La deserción escolar en el nivel medio superior (CASO IPN). México: Ciesas.

INEE (2017a). Directrices para mejorar la permanencia escolar en la educación media superior. México.

INEE (2017b). La educación obligatoria en México. Informe 2017. México.

INEE (2017c). Planea. Evaluaciones de logro referidas al sistema educativo nacional. Último grado de educación media superior, ciclo escolar 2016-2017. México.

INEE (2017d). Planea. Resultados nacionales 2017. Educación media superior. México.

INEE (2016). Banco de indicadores educativos (BIE). México.

INEGI (2015). Encuesta Intercensal 2015. Base de datos. México.

INEGI (2009). Encuesta Nacional de Ocupación y Empleo (ENOE). México. 
Instituto Internacional de Planeamiento de la Educación de la Organización de las Naciones Unidas para la Educación, la Ciencia y la Cultura (IIPE-Unesco). (2010). Evaluación del Programa Construye T. Informe final de la evaluación de procesos. Nueva York.

Instituto Mexicano de la Juventud y Secretaría de Educación Pública (2010). Encuesta Nacional de Juventud. México.

Miranda, F. (2013). La evaluación a debate entre la calidad y la desafiliación de los jóvenes de la educación secundaria en América Latina. Revista Latinoamericana de Educación Comparada, vol. 4, núm. 4, pp. 41-58. Recuperado de http://www.saece.com.ar/relec/revistas/4/mon3.pdf

Miranda, F. (2012a). Ambientes escolares. Documento de trabajo. México.

Miranda, F. (2012b). Los jóvenes contra la escuela. Un desafío para pensar las voces y tiempos para América Latina. Revista Latinoamericana de Educación Comparada, vol. 3, núm. 3, pp. 71-84. Recuperado de http://www.saece.com. ar/relec/revistas/3/art6.pdf

Miranda, F. e Islas, J. (2016). Los jóvenes, sus derechos y la equidad en la educación media superior en México: hacia una agenda de política educativa. Polifonías, Revista de Educación, vol. 5, núm. 9, pp. 149-177. Recuperado de http:// www.polifoniasrevista.unlu.edu.ar/sites/www.polifoniasrevista.unlu.edu. ar/files/site/9\%20Miranda\%20e\%20Islas.pdf

Mockus, S. (2010). Anfibios culturales y divorcio entre ley, moral y cultura. $R e$ vista Aleph, núm. 153, pp. 7-14.

Montgomery, M. \& Hewett, P. (2005). Urban poverty and health in developing countries: Household and neighborhood effects. Demography, vol. 42, núm. 3, pp. 397-425. https://doi.org/10.1353/dem.2005.0020

Morduchowicz, A. (2003). Los costos de la obligatoriedad escolar en el nivel medio. En E. Tenti (ed.). Educación media para todos. Los desafíos de la democratización del acceso (pp. 65-84). Argentina: Unesco/IIPE/Altamira/OSDE.

Quevedo, L. (2003). La escuela frente a los jóvenes, los medios de comunicación y los consumos culturales en el siglo XXI. En E. Tenti (ed.). Educación media para todos. Los desafíos de la democratización del acceso (pp. 139-157). Argentina: Unesco/IIPE/Altamira/OSDE.

Reguillo, R. (2000). Emergencia de culturas juveniles: estrategias del desencanto. Bogotá, Colombia: Grupo Editorial Norma.

Ruel, M., Haddad, L. \& Garrett, J. (2008). Rapid urbanization and the challenges of obtaining food and nutrition security. En R. D. Semba \& M. W. Bloem (eds.). Nutrition and health in developing countries (pp. 465-482). Totowa, NJ: Humana Press.

SEP (2017). Modelo educativo para la educación obligatoria. Educar para la libertad y la creatividad. Ciudad de México.

SEP (2013). Programa Sectorial de Educación 2013-2018. México.

SEP e Instituto Nacional de Salud Pública (2015). Análisis del movimiento contra el abandono escolar en la educación media superior. Recuperado de http:// www.sems.gob.mx/work/models/sems/Resource/11390/6/images/reporte_abandono.pdf SEP y Consejo para la Evaluación de la Educación del Tipo Medio Superior (2012). Reporte de la Encuesta Nacional de Deserción en la Educación Media Superior. México. Recuperado de http://www.sems.gob. mx/work/models/sems/Resource/10787/1/images/Anexo_6Reporte_de_ la_ENDEMS.pdf 
Solís, P. (2018). La transición de la secundaria a la educación media superior en México: el difícil camino a la cobertura universal. Perfiles Educativos, vol. XI, núm. 159, pp. 66-89. Recuperado de https://www.researchgate.net/publication/323817415_La_transicion_de_la_secundaria_a_la_educacion_media_superior_en_Mexico_El_dificil_camino_a_la_cobertura_universal

Székely, M. (2015). Estudio sobre los principales resultados y recomendaciones de la investigación y evaluación educativa en el eje de prevención y atención a la deserción escolar en educación media superior (documento interno). México: INEE.

Tenti, E. (ed.). (2003). Educación media para todos. Los desafíos de la democratización del acceso. Argentina: Unesco/IIPE/Altamira/OSDE.

Tinto, V. (1989). Definir la deserción: una cuestión de perspectiva. Revista de la Educación Superior, vol. 18, núm. 71, pp. 1-9.

Tinto, V. (1982). Limits of theory and practice in student attrition. The Journal of Higher Education, vol. 53, núm. 6, pp. 687-700.

Tinto, V. (1975). Dropout from higher education: A theoretical synthesis of recent research. Review of Educational Research, vol. 45, núm. 1, pp. 89-125.

UNAM (2016). Seminario de Investigación en Juventud de la Universidad Nacional Autónoma de México. Evaluación de la política contra la interrupción escolar en la Educación Media Superior (documento interno). México.

Unesco (2017). La Unesco: trabajando con y para los jóvenes. París, Francia.

Unión Europea (s.f.). Europa 2020: la estrategia de la Unión Europea para el crecimiento y la ocupación. Bruselas, Bélgica.

Valora, V. (2016). Intervención para abatir el abandono escolar en educación media superior en México: diseño, operación y resultados de la estrategia "Yo no abandono", desde la mirada de los actores escolares y autoridades educativas 2016 (documento interno). Ciudad de México: Instituto Belisario Domínguez.

Weiss, E. (2015). El abandono escolar en la educación media superior: dimensiones, causas y políticas para abatirlo. En R. Ramírez Raymundo (ed.). Desafíos de la educación media superior (pp. 81.160). Ciudad de México: Instituto Belisario Domínguez. 